\title{
PERSISTENCE OF HEPATOCELLULAR CARCINOMA RISK IN HEPATITIS C PATIENTS WITH A RESPONSE TO IFN AND CIRRHOSIS REGRESSION 17
}

Roberta D’Ambrosio ${ }^{1}$, Alessio Aghemo ${ }^{2,3}$, Maria Grazia Rumi ${ }^{4}$, Elisabetta Degasperi ${ }^{1}$, Angelo Sangiovanni ${ }^{1}$, Marco Maggioni ${ }^{5}$, Mirella Fraquelli ${ }^{6}$, Riccardo Perbellini ${ }^{1}$, William Rosenberg ${ }^{7}$, Pierre Bedossa $^{8}$, Massimo Colombo ${ }^{3}$, Pietro Lampertico ${ }^{1}$

${ }^{1}$ A.M. and A. Migliavacca Center for Liver Disease, Division of Gastroenterology and Hepatology, Fondazione IRCCS Cà Granda Ospedale Maggiore Policlinico, Università degli Studi di Milano, Milan, Italy. ${ }^{2}$ Humanitas University Department of Biomedical Sciences, Rozzano-Milan, Italy. ${ }^{3}$ Humanitas Clinical and Research Center, Rozzano-Milan, Italy. ${ }^{4}$ Division of Hepatology, Ospedale San Giuseppe, Università degli Studi di Milano, Milan, Italy. ${ }^{5}$ Division of Pathology, Fondazione IRCCS Cà Granda Ospedale Maggiore Policlinico, Università degli Studi di Milano, Milan, Italy. ${ }^{6}$ Division of Gastroenterology and Endoscopy, Fondazione IRCCS Cà Granda Ospedale Maggiore Policlinico, Università degli Studi di Milano, Milan, Italy. ${ }^{7}$ Center for Hepatology, Division of Medicine, University College of London, London, United Kingdom. ${ }^{8}$ Department of Pathology and INSERM U773, Hopital Beaujon, Universitée Paris-Diderot, Clichy, France. 


\section{Corresponding author}

Roberta D'Ambrosio, MD

Division of Gastroenterology and Hepatology

Fondazione IRCCS Ca' Granda Ospedale Maggiore Policlinico

Università degli Studi di Milano

Via F. Sforza 35

20122 Milano

Italy

Tel. 0039-0255035432

Fax. 0039-0250320410

E-mail: roberta.dambrosio@policlinico.mi.it

\section{Text word count: $\underline{4.399}$}

Number of figures and tables: 5 Tables +2 Figures $+\underline{2 \text { Supplementary Tables }}$

\section{List of abbreviations}

DAA: direct-acting antivirals; $\mathrm{HCV}$ : hepatitis $\mathrm{C}$ virus; IFN: interferon; $\mathrm{HCC}$ : hepatocellular carcinoma; SVR: sustained virological response; LB: liver biopsy; US: ultrasound; HBV: hepatitis B virus; HIV: human immunodeficiency virus; ALT: alanine aminotransferase; $\gamma \mathrm{GT}$ : $\gamma$ glutamil transferase; HDL: high density lipoprotein; $\alpha \mathrm{FP}$ : alpha-fetoprotein; CK7: citokeratine-7;GS: glutamine synthetase; CYP2E1: cytochrome P450 2E1; $\alpha$ SMA: $\alpha$-smooth muscle actin; TE: transient elastography; LSM: liver stiffness measurement; NIT: non invasive tests; GI: gastrointestinal; HR: hazard ratio; CI: confidence interval; BMI: body mass index; ULN: upper limit of normal; RFTA: radiofrequency thermo-ablation. 


\section{Statement of interests}

Competing Interests: Prof. William Rosenberg has been paid by Siemens for providing lectures on ELF marker. He is named inventor on a patent wholly owned by Siemens. He has no financial competing relevant interests to declare.

\section{Financial support}

Nothing to declare 


\section{ABSTRACT}

Background and Aim: In patients with HCV-related cirrhosis, a SVR may lead to cirrhosis regression. Whether histological changes translate into prevention of long-term complications, particularly hepatocellular carcinoma (HCC) is still unknown. This was investigated in a cohort of histological cirrhotics who had been prospectively followed-up for 10 years after the achievement of a SVR to IFN. Methods: 38 SVR cirrhotics who underwent a liver biopsy (LB) 5 years postSVR were prospectively followed to assess the impact of cirrhosis regression on clinical endpoints. Results: During a follow-up of 86 (30-96) months from LB, no patients developed clinical decompensation, whilst 5 (13\%) developed HCC after 79 (7-88) months. The 8-year cumulative probability of $\mathrm{HCC}$ was $17 \%$, without differences between patients with or without cirrhosis regression [19\% (95\% CI 6-50\%) vs. 14\% (95\% CI 4-44\%), p=0.88]. Patients who developed or did not an HCC had similar rates of residual cirrhosis $(p=1.0)$, collagen content $(p=0.48)$, METAVIR activity $(\mathrm{p}=0.34)$, portal inflammation $(\mathrm{p}=0.06)$ and steatosis $(\mathrm{p}=0.17)$. At baseline, patients who developed an HCC had higher $\gamma$ GT (HR 1.03, 95\% CI 1.00-1.06; $\mathrm{p}=0.014)$ and glucose (HR 1.02, 95\% CI 1.00-1.02; $\mathrm{p}=0.012$ ) values; moreover, they had increased Forns Score (HR 12.8, 95\% CI 1.14-143.9; $\mathrm{p}=0.039)$, Lok Index (HR 6.24, 95\% CI 1.03-37.6; $\mathrm{p}=0.046)$ and PLF (HR 19.3, 95\% CI 1.72-217.6; $\mathrm{p}=0.016$ ) values. One regressor died of lung cancer. The 8-year cumulative survival probability was $97 \%$, independently on cirrhosis regression $(96 \%$ vs. $100 \%, \mathrm{p}=1.0)$ or HCC $(100 \%$ vs. $97 \%, \mathrm{p}=1.0)$. Conclusions: Post-SVR cirrhosis regression does not prevent HCC occurrence.

\section{Key-words}

Sustained virological response (SVR), cirrhosis regression, hepatocellular carcinoma (HCC), liver biopsy, non-invasive tests (NITs), transient elastography (TE)

\section{Abstract word Count: 250}




\section{Bullet Points}

- Cirrhotic patients who achieve an SVR to anti-HCV regimens should remain on regular HCC surveillance since it has been demonstrated that the risk of liver cancer is not fully abrogated by viral eradication

- Whether regression of cirrhosis following an SVR may prevent liver-related complications is still unknown

- In our cohort of cirrhotic patients who achieved an SVR through IFN-based regimens, HCC occurred at low rates independently on post-SVR cirrhosis regression

- $\quad$ Neither clinical parameters, post-SVR histological features or non-invasive tests were able to predict the occurrence of $\mathrm{HCC}$ in our cohort of selected patients 


\section{INTRODUCTION}

The advent of safe and effective direct-acting antivirals (DAA) has revolutionized treatment of chronic hepatitis $\mathrm{C}$ raising the bar of virus eradication above $90 \%$ in hepatitis $\mathrm{C}$ virus (HCV) cirrhotics who have longer been the hardest patients to be cured with interferon (IFN) ${ }^{\mathbf{1}}$. In the IFN era, patients with advanced liver disease who achieved HCV eradication appeared to be partially protected against the risk of clinical decompensation and hepatocellular carcinoma (HCC) development. The identification of risk factors associated with HCC development in HCV cirrhotics with a sustained virological response (SVR) could allow to design individualized surveillance schedules, hence containing healthcare costs. In a single-center study in France, end-stage complications of $\mathrm{HCV}$ were fully prevented in the subgroup of SVR patients with histologically

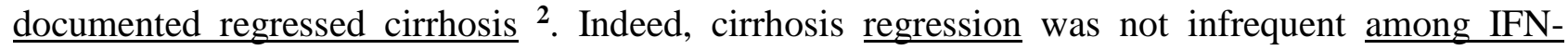
responders, with rates from $24 \%$ to $100 \%{ }^{2-16}$.

Mitigating the impact of cirrhosis regression in SVR patients, however, is suboptimal accuracy of liver biopsy (LB) to establish cirrhosis regression, owing to the $25 \%$ risk of fibrosis misclassification of small liver tissue cores ${ }^{17}$. This coupled with the fact that the French study correlated the histological regression of cirrhosis with combined endpoints including liver failure, bleeding and HCC, prompted us to prospectively evaluate the clinical outcome of a cohort of 38 cirrhotics, $23(61 \%)$ of whom with histological cirrhosis regression 5 years after the achievement of an SVR ${ }^{18}$. Regressors and non regressors were then subjected to surveillance for HCC with 6month abdominal ultrasound (US). 


\section{MATERIAL AND METHODS}

\section{Patient population}

This is a long-term follow-up study of previously published Italian-French cooperative study conducted on $38 \mathrm{HCV}$ cirrhotics who underwent paired LB, before and after the achievement of an SVR to IFN-based regimens ${ }^{\mathbf{1 8}}$. All patients were prospectively followed-up after post-SVR LB (baseline); hepatitis B virus (HBV) or human immunodeficiency virus (HIV) coinfections as well as alcohol consumption, which were previously excluded ${ }^{\mathbf{1 8}}$, were confirmed during follow-up. Patients underwent 6-month clinical follow-up with blood test and US. Data entry was completed on 31 March 2017. Informed consent was obtained from each patient included in the study. The protocol was approved by the Institutional Board of our Department (Ethical Committee Milan Area 2) and conforms to the ethical guidelines of the 1975 Declaration of Helsinki.

\section{Biochemical assays}

The following normal values were used: alanine aminotransferase (ALT) $<19$ U/1 for females and $<30 \mathrm{U} /$ f for males $^{19}$; $\gamma$ glutamil-transferase $(\gamma \mathrm{GT})<36 \mathrm{U} / 1$ for females and $<60 \mathrm{U} / 1$ for males; cholesterol <200 mg/dL, high density lipoprotein (HDL) cholesterol >60 mg/dL; triglycerides < 150 $\mathrm{mg} / \mathrm{dL} ;$ alpha-fetoprotein $(\alpha \mathrm{FP})<7 \mu \mathrm{g} / \mathrm{ml}$.

\section{Histological assessment}

Post-SVR LB were performed with $16 \mathrm{G}$ Menghini-like semi-automatic needles ${ }^{\mathbf{1 8}}$. Post-treatment fibrosis was staged according to the METAVIR scoring system ${ }^{\mathbf{2 0}}$, and cirrhosis regression was defined as previously published as METAVIR $<$ F4 ${ }^{\mathbf{1 8}}$. Residual fibrosis was quantitatively assessed (\%) by morphometry. Residual activity was classified according to both METAVIR and Ishak classifications ${ }^{\mathbf{2 1 , 2 2}}$, and immunohistochemistry [citokeratine-7 (CK7), glutamine synthetase (GS), 
cytochrome P450 2E1 (CYP2E1), $\alpha$-smooth muscle actin ( $\alpha$ SMA), and CD34] was carried-out semi-quantitatively using a three grade system $(0,1,2){ }^{\mathbf{1 8}}$ (see results). When possible, histology was obtained to confirm HCC, as well as from extra-lesion tissue.

\section{Non-invasive assessment of fibrosis}

Patients underwent post-SVR non-invasive assessment of residual liver fibrosis ${ }^{\mathbf{2 3 , 2 4}}$, which was concomitant to LB. Transient elastography (TE) was performed as already described ${ }^{25}$, liver stiffness measurement (LSM) being expressed in kilopascal $(\mathrm{kPa})$. The following serological indirect and direct markers of liver fibrosis (Non Invasive Tests, NITs) were tested according to their formula, as previously published ${ }^{24}$ : APRI, CDS, FIB-4, FibroQ, Forns Score, GUCI, King's Score, Lok Index, PLF, and ELF. Reference cut-offs for cirrhosis in viremic patients were APRI > 1.5, $\mathrm{CDS}>8, \mathrm{FIB}-4>3.25$, FibroQ $>2.6$, Forns Score $>6.9$, GUCI > 0.26, King's Score $>16.7$, Lok Index > 0.5 and PLF > 2.98 (Supplementary Table 1).

\section{Study endpoints}

The primary endpoint of the study was the relationship between post-SVR cirrhosis regression and the risk of developing liver-related events [i.e. HCC, liver failure or varices-related gastrointestinal (GI)-bleeding]. Hepatocellular carcinoma was diagnosed according to international criteria ${ }^{\mathbf{2 6 - 2 9}}$. Liver failure was defined as an episode of ascites, jaundice or hepatic encephalopathy. In case multiple events occurred in a single subject, only the first event contributed to outcome measure.

Secondary and tertiary endpoints were the relationship between other post-SVR histological features as well as non-invasive tests (TE and NITs), and the risk of liver-related complications. 


\section{Statistical analysis}

In all patients, follow-up started at the time of post-SVR LB (baseline) $\mathbf{1 8 , 2 3 , 2 4}^{\mathbf{2}}$ and patients were censored at the time of their first liver-related complication or at their last follow-up date.

Categorical variables were reported as frequencies (percentages) and continuous variables as median (range). Categorical variables were compared using the $\chi 2$ or the Fisher's exact tests; continuous variables were compared using the Student t-test, the Mann-Whitney $U$-test or the Kruskall-Wallis test, when appropriate. All tests were two-sided and used a significance level of 0.05 .

The Kaplan-Meier method was used to assess the cumulative incidence of clinical events during follow-up. Cox regression analysis was used to identify baseline variables associated with HCC during follow-up. Variables with a threshold value of $<0.05$ were considered statistically significant. Results are expressed as adjusted hazard ratio (HR) and their $95 \%$ confidence intervals (C.I.). Data handling and analysis were performed with StataView package (SAS Institute Inc., Cary, NC).

\section{RESULTS}

Patients included in the study were males (65\%), with a median age of 66 (46-75) years; SVR was achieved 61 (48-104) months before. Prevalence of BMI >25 (55\%), diabetes (10.5\%), hypercholesterolemia (55\%) and hypertriglyceridemia (13\%) (Table 1) were similar to those recorded at the time of SVR achievement (p-values 0.36, 0.36, 0.62 and 1.0, respectively). Cirrhosis regression was documented in $23(61 \%)$ patients (Table 1). Patients with and without cirrhosis regression were similar in terms of demographic and clinical features (Table 1), although $\underline{\text { F4 }}$ patients had a higher prevalence of hypertriglyceridemia $(\mathrm{p}=0.07)$ and higher levels of fasting glucose $(\mathrm{p}=0.08)$, which however did not reach statistical significance. Moreover, patients with 
cirrhosis regression had lower LSM values when compared to non regressors $(9.1 \mathrm{kPa}$ vs. $12.9 \mathrm{kPa}$, $\mathrm{p}=0.004)$ (Table 1), but were similar according to most of the NITs (Supplementary Table 2)

After LB, all patients were adherent to 6-month surveillance, and were followed-up for 86 (30-96) months, without any differences between patients with and without cirrhosis regression [88 (74-96) vs. 83 (30-95), $\mathrm{p}=0.52$ ]. During follow-up, most of them showed persistently normal values of both ALT and $\gamma \mathrm{GT}$ (71\% and 68\%, respectively).

\section{Liver-related events}

No episodes of clinical decompensation or GI-bleeding were recorded, whilst HCC developed in 5 (13\%) patients. Median time to HCC development was 79 (7-88) months. The 8-year cumulative probability of $\mathrm{HCC}$ was $17 \%$ (95\% CI: $7 \%$ - 39\%), with an annual estimated incidence rate of $1.2 \%$

\section{(Figure 1A).}

At the time of HCC development, patients (80\% males) had a median age of 71 (62-74) years, with BMI value of 26 (25-28). Most of them had persistently normal ALT and $\gamma \mathrm{GT}$ values (80\% and $60 \%$, respectively). Diabetes was present in 2 (40\%), hypercholesterolemia in $3(60 \%)$ and hypertriglyceridemia in $1(20 \%)$ of them. All patients had compensated liver diseases (CPT A5), with a median $\underline{\text { LSM }}$ of 9.3 (8.5-36.3), without differences between patients with and without cirrhosis regression $[\mathrm{F}<4$ vs. F4: 9.3 (5.4-36.6) vs. 10.2 (8.5-11.8), p=1.0]. Alpha-fetoprotein was normal in most of them (80\%), with a median value of $4.2(2.6-57) \mathrm{ng} / \mathrm{ml}$. In all cases, HCC was single, sized 22 (19-30) mm, non-metastatic; therefore curative approaches were offered (4 RFTA, 1 resection). Histology was available for three patients at the time of $\mathrm{HCC}$ treatment (Figure 2), which confirmed post-SVR LB fibrosis stage (2 F3, 1 F4) (see below).

At baseline, patients who developed or did not develop a HCC were similar according to the most important demographic and clinical features, although the formers displayed higher $\gamma \mathrm{GT}$ values 
$(\mathrm{p}=0.04)$ and an increased prevalence of diabetes $(\mathrm{p}=0.07)$ (Table 2). No differences in HCC development were observed in patients with and without esophageal varices at baseline $(13 \%$ vs. $\underline{25 \%, \mathrm{p}=0.49)}$

At univariate analysis, fasting glucose (HR 1.02, 95\% CI 1.00-1.02; p=0.012) and $\gamma \mathrm{GT}$ values (HR 1.03, 95\% CI 1.00-1.06; $\mathrm{p}=0.014)$ were significantly associated with HCC development, whereas diabetes (HR 5.62, 95\% CI 0.93-33.9; $\mathrm{p}=0.06$ ) and steatosis (HR 0.99, 95\% CI: 1.05-0.09; $\mathrm{p}=0.092$ ) were close to statistical significance (Table 3).

Patients who did develop an HCC were also similar to those who did not in most of the baseline histological features, as assessed at the time of post-SVR LB. In patients who developed HCC advanced stages of residual fibrosis were frequent $(\mathrm{p}=0.06)$ although the prevalence of cirrhosis was similar in patients with or without HCC (40\% vs. 40\%, $\mathrm{p}=1.0)$ (Table 4). The 8-year cumulative probability of HCC was similar in patients with or without cirrhosis regression $(\mathrm{p}=0.88)$. However, the two patients with residual cirrhosis developed HCC earlier than those who achieved cirrhosis regression (7 and 27 months vs. 79, 80 and 88 months from LB) (Figure 1B). In addition, no differences were observed in terms of steatosis, residual collagen content or improvement in the area of fibrosis as compared to pre-treatment values. Similarly, immunohistochemistry did not differ between the two groups.

Baseline LSM did not differ among patients who subsequently developed or not an HCC (12.6 kPa vs. $9.8 \mathrm{kPa}, \mathrm{p}=0.78$ ) (Table 2). Nevertheless, although we did not observe any difference for most of the NITs according to the presence of HCC, patients who developed liver cancer had higher postSVR values of Lok Index ( $\mathrm{p}=0.05)$, whilst Forns Score $(\mathrm{p}=0.09)$ and PLF $(\mathrm{p}=0.06)$ were close to statistical significance (Table 5). At univariate analysis, the following NITs were associated with an increased risk of HCC: Forns Score > $6.9(\mathrm{p}=0.039)$, Lok Index > $0.5(\mathrm{p}=0.046)$ and PLF $>2.98$ $(\mathrm{p}=0.016)$ (Table 3). 


\section{Non-liver related events}

Extra-hepatic malignancies were the only non-liver related events recorded in $3(7.8 \%)$ patients, all without HCC. Uterine cancer, lung cancer and rectal cancer developed after 71 (51-76) months from baseline.

\section{Survival}

During 86 (30-96) months after LB, one patient died of lung cancer (71 months). The 8-year cumulative survival was $97 \%$, and was not influenced by cirrhosis regression $(96 \%$ vs. $100 \%$, $\mathrm{p}=1.0)$ or HCC development (100\% vs. 97\%, $\mathrm{p}=1.0)$. Post-LB follow-up was similar in patients who did develop or not an HCC [HCC 90 (30-96) vs. non-HCC 86 (74-95) months, p=0.70].

One patient was lost during follow-up, 3 months after HCC treatment (RFTA), i.e. 30 months after post-SVR LB.

\section{DISCUSSION}

This prospective study demonstrates that the risk of $\mathrm{HCC}$ was not fully abrogated in patients who, following an SVR to IFN, had histologically documented regression of cirrhosis, which was defined according to previously published studies $\mathbf{4 , 5 , 1 2 , 1 8}$. Cirrhosis regression was documented in $61 \%$ of patients in our cohort after a median follow up of 5-year post-SVR ${ }^{\mathbf{1 8}}$, whereas in the subsequent follow-up of 86 months liver cancer was the only liver-related complication. The fact that patients who had cirrhosis at the onset of IFN-based therapies face a life-long, residual risk of developing HCC after achieving an SVR, is well documented ${ }^{\mathbf{3 0 , 3 1}}$. In our study, despite the small sample size of the cohort and the low incidence of HCC, the analysis of the correlation between histological and clinical features and risk of HCC during a 10-year follow-up, showed that, at variance with the 
French study, cirrhosis regression did not prevent HCC development ${ }^{2}$. In that study, the only adverse outcomes among SVR patients with residual cirrhosis were three cases of HCC and one GIbleeding. This is not a trivial point, since our findings contradict expert recommendations that suggest to drop surveillance in SVR patients when post-SVR cirrhosis regression is documented ${ }^{32,33}$, at the same time reinforcing AASLD and EASL recommendation for continued surveillance of SVR patients, independently from cirrhosis regression ${ }^{1,34}$.

Not unexpectedly, the rates of HCC in our SVR patients were quite low (1.2\%) and similar in both regressors and non regressors $(\mathrm{p}=0.88)$, telling us that the dissociation between HCC risk and cirrhosis regression supports the need for life-long surveillance in SVR patients with a diagnosis of cirrhosis before antiviral therapy. At the time of surgical treatment of HCC, cirrhosis regression could be further confirmed histologically.

Interestingly, patients with and without HCC were similar for most of the histological features assessed at post-SVR LB, thus preventing us to find any characteristics associated with an increased risk of liver cancer after virus eradication. Of note, HCC was not predicted by persistence of steatosis, residual portal inflammation and collagen content amount. Moreover, we found no differences according to several immunohistochemical markers, when comparing patients who did or did not develop a HCC, thus suggesting that persistence of most of the microscopic features of cirrhosis (i.e. sinusoidal capillarization, metabolic zonation or hepatic stellate cells activation) have no role in favouring liver cancer development. This is a novelty, since to the best of our knowledge no studies have investigated the correlation between immunohistochemical markers and the risk of liver-complication among SVR patients.

Apart from histological findings, while patients maintained persistently normal values of serum transaminases and $\gamma \mathrm{GT}$ with respect to pre-treatment, HCC mostly occurred in patients with metabolic syndrome, including diabetes. We previously reported an inverse relationship between 
$\gamma$ GT values and rates of cirrhosis regression after the achievement of an SVR ${ }^{\mathbf{1 8}}$. Our findings are in line with previous studies in HCV patients with an SVR to IFN ${ }^{35-37}$ where metabolic disorders, including diabetes, were associated with a residual risk of $\mathrm{HCC}$.

Finally, we correlated post-SVR NITs values with the residual risk of HCC in our cohort and found that patients who developed a liver cancer displayed higher baseline values of Forns Score, Lok Index and PLF. Among them, Forns Score includes $\gamma \mathrm{GT}$, age and cholesterol which have been previously demonstrated to be associated with an increased risk of HCC after an SVR. This is an important finding, since scarce data exist on the correlation between post-SVR NIT values and residual risk of HCC in non-viremic patients ${ }^{38,39}$. Different serum biomarkers have been retrospectively analyzed, in all cases without any correlation with histology, and studies provided discordant results. In fact, Toyoda and colleagues ${ }^{38}$ found that low pre-treatment Forns Score values were protective against the risk of liver cancer, whereas none of the 20 tests analyzed in the study by Thandassery et al. reached any significant predictive value at multivariate analysis ${ }^{\mathbf{3 9}}$. On the contrary, we found that Forns Score, Lok Index and PLF values above the viremic F4 cut-off were associated with an increased risk of HCC. Although these data have been obtained in a much selected cohort, thus preventing us from assessing NITs real predictive values, we think that this aspect need further external large validation.

We acknowledge that our study does have some limitations related to our selection criteria and the small number of end points, i.e. HCC. The latter, however, is the inevitable consequence of prospectively investigating the cohort composed by IFN-cured cirrhotics who accepted to repeat a liver biopsy after achieving a SVR, an event that reduces the risk of HCC. Noticeably, compliance with the study protocol was optimal as one patient was lost during follow-up, only and this happened after achieving HCC diagnosis. Finally, although the definition of cirrhosis regression we used is constraining and we could not exclude sampling errors $\mathbf{4 0}$, we did refer to the same criteria 
used in our previously published studies $\mathbf{1 8 , 2 3 , 2 4}$, which however have been widely accepted in most of the other studies demonstrating cirrhosis and fibrosis regression among $\underline{\text { SVR patients treated with }}$ IFN 4,5,12. Whether a less restrictive definition of cirrhosis regression should be used when analyzing the relationship between residual fibrosis and liver-related events could be matter of debate. In fact, in our study no HCC were observed among patients staged F1-F2 (i.e. METAVIR decrease $>1$ stage), thus suggesting that a less stringent criteria to define cirrhosis regression could be useful in identifying those patients more likely to remain free from liver cancer. This would be in line with what previously reported in the French study by Mallet and colleagues ${ }^{2}$.

In conclusion, the finding that HCC developed also in $\underline{\mathrm{SVR}}$ patients with cirrhosis regression $\underline{(\mathrm{F} 3)}$ greatly attenuates the need for refining the management of SVR patients in relation to residual liver fibrosis. The fact that regressed patients developed HCC later than non regressors deserves attention, as it might suggest the presence of causes of liver disease progression other than $\mathrm{HCV}$. However, two out of three patients with a liver cancer showed regression to METAVIR F3 in the absence of any additional cause of liver disease. We wish to think that the risk of HCC in such regressors is the consequence of long lasting exposure of liver cells to direct and indirect carcinogenetic effects of $\mathrm{HCV}$, as clearly documented in more than one experimental study $\mathbf{4}$.

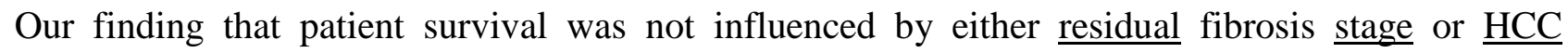
occurrence (one patient died of non-liver related complication, only) is in line with a recently published multicentre study in Italy ${ }^{\mathbf{3 0 , 3 1}}$ where $\underline{\text { SVR }}$ cirrhotics showed comparable survival rates as the general population ${ }^{31}$.

We acknowledge that our study was to some extent weakened by the strict selection criteria we adopted; yet, at the same time it provides robust information to refine surveillance of SVR patients, i.e. not to interrupt surveillance in SVR patients with an initial diagnosis of cirrhosis. 
In conclusion, our finding that the risk of liver cancer among SVR cirrhotics was not fully abrogated in regressed patients fully supports the recommendation of international societies for lifelong surveillance strategies, independently on the stage of post-SVR liver disease. Although deserving further validation, the finding of persistently high post-SVR values of certain NITs in patients with liver cancer might be useful to tailor surveillance strategies in cured patient, independently on histological assessment of residual fibrosis. At the moment, our results reinforce futility of repeated liver biopsies and investigations with non-invasive tests in SVR patients lacking other than HCV risk factors for liver disease.

\section{Acknowledgments}

The authors thank Ms Anna Molinari for her expert secretarial assistance. 


\section{FIGURE LEGEND}

Figure 1. Cumulative probability of $\mathrm{HCC}$ in the entire cohort (1A) and according to residual fibrosis stage (1B).

Figure 2. Resected liver specimen from a patient who developed an HCC 80 months after postSVR LB (i.e. 180 months after SVR) showing subcapsular well-demarcated nodule (A; H\&E 5x), corresponding to moderately differentiated trabecular HCC (B; H\&E 200x); non-tumoral liver parenchyma demonstrates largely incomplete nodular structure $(\mathrm{C}$; Masson's Trichrome $5 \mathrm{x})$ with portal-to-portal complete and incomplete fibrous septa and peri-septal steatosis, without necroinflammatory activity (D; Masson's Trichrome 5x). 


\section{REFERENCES}

1. European Association for the Study of the Liver. EASL recommendations on treatment of hepatitis C 2016. J Hepatol 2017;66:153-194

2. Mallet V, Gligenkrantz H, Serpaggi J, et al. Brief communication: the relationship of regression of cirrhosis to outcome in chronic hepatitis C. Ann Intern Med 2008;149:399-403

3. Reichard O, Glaumann H, Frydén A, Norkrans G, Wejstal, Weiland O. Long-term follow-up of chronic hepatitis $\mathrm{C}$ patients with sustained virological response to alpha-interferon. J Hepatol 1999;30:783-787

4. Shiratori Y, Imazeki F, Moriyama M, et al. Histological improvement of fibrosis in patients with hepatitis $\mathrm{C}$ who have sustained response to interferon therapy. Ann Intern Med 2000;132:517-524

5. Poynard T, McHutchison J, Manns M, et al. Impact of pegylated interferon alfa-2b and ribavirin on liver fibrosis in patients with chronic hepatitis C. Gastroenterology 2002;122:13031313

6. Arif A, Levine RA, Sanderson SO, et al. Regression of fibrosis in chronic hepatitis C after therapy with interferon and ribavirin. Dig Dis Sci 2003;48:1425-1430

7. Pol S, Carnot F, Nalpas B, et al. Reversibility of hepatitis C-related cirrhosis. Hum Pathol 2004;35:107-112

8. Yoshida H, Tateishi R, Arakawa Y, et al. Benefit of interferon therapy in hepatocellular carcinoma prevention for individual patients with chronic hepatitis C. Gut 2004;53:425-430

9. $\mathrm{Yu}$ ML, Lin SM, Chuang WL, et al. A sustained virological response to interferon or interferon/ribavirin reduces hepatocellular carcinoma and improves survival in chronic hepatitis C: a nationwide, multicenter study in Taiwan. Antivir ther 2006;11:985-994 
10. Bruno S, Stroffolini T, Colombo M, et al. Sustained virological response to interferon-alpha is associated with improved outcome in HCV-related cirrhosis: a retrospective study. Hepatology 2007;45:579-587

11. Veldt BJ, Heathcote EJ, Wedemeyer H, et al. Sustained virological response and clinical outcomes in patients with chronic hepatitis $\mathrm{C}$ and advanced fibrosis. Ann Intern Med 2007;147:677-684

12. Everson GT, Balart L, Lee SS, et al. Histological benefits of virological response to peginterferon alfa-2a monotherapy in patients with hepatitis $\mathrm{C}$ and advanced fibrosis or compensated cirrhosis. Aliment Pharmacol Ther 2008;27:542-551

13. George SL, Bacon BR, Brunt EM, Mihindukulasuriya KL, Hoffmann J, Di Bisceglie AM. Clinical, virologic, histologic and biochemical outcomes after successful HCV therapy: a 5year follow-up of 150 patients. Hepatology 2009;49:729-738

14. Morgan TR, Ghany MG, Kim HY, et al. HALT-C Trial Group. Outcome of sustained virological responders with histologically advanced chronic hepatitis C. Hepatology 2010;52:833-844

15. Cardoso AC, Moucari R, Figueiredo-Mendes $\mathrm{C}$, et al. Impact of peginterferon and ribavirin therapy on hepatocellular carcinoma: incidence and survival in hepatitis $\mathrm{C}$ patients with advanced fibrosis. J Hepatol 2010;52:652-657

16. Van der Meer AJ, Veldt BJ, Feld JJ, et al. Association between sustained virological response and all-cause mortality among patients with chronic hepatitis $\mathrm{C}$ and advanced hepatic fibrosis. JAMA 2012;308:2584-2593

17. Bedossa P, Dargere D, Paradis V. Sampling variability of liver fibrosis in chronic hepatitis CHepatology 2003;38:1449-1457. 
18. D'Ambrosio R, Aghemo A, Rumi MG, et al. A morphometric and immunohistochemical study to assess the benefit of a sustained virological response in hepatitis $\mathrm{C}$ virus patients with cirrhosis. Hepatology 2012;56:532-543

19. Prati D, Taioli E, Zanella A, et al. Updated definitions of healthy ranges for serum alanine aminotransferase levels. Ann Intern Med 2002;137:1-10

20. The METAVIR cooperative group. Inter- and intra-observer variation in the assessment of liver biopsy of chronic hepatitis C. Hepatology 1996;20:15-20

21. Ishak K, Baptista A, Bianchi L, et al. Histological grading and staging of chronic hepatitis. J Hepatol 1995;22:696-699

22. Bedossa P, Poyard T. An algorithm for the grading of activity in chronic hepatitis C. Hepatology 1996;24:289-293

23. D’Ambrosio R, Aghemo A, Fraquelli M, et al. The diagnostic accuracy of Fibroscan ${ }^{\circledR}$ for cirrhosis is influenced by liver morphometry in HCV patients with a sustained virological response. J Hepatol 2013;59:251-256

24. D’Ambrosio R, Degasperi E, Aghemo A, et al. Serological tests do not predict residual fibrosis in hepatitis $\mathrm{C}$ cirrhotics with a sustained virological response to Interferon. PLoS One 2016;11:e0155967

25. Sandrin L, Fourquet B, Hasquenoph JM, et al. Transient elastography: a new non-invasive method for assessment of hepatic fibrosis. Ultrasound Med Biol 2003;29:1705-1713

26. Bruix J, Sherman M, Llovet JM, et al. Clinical management of hepatocellular carcinoma. Conclusion of the Barcelona 2000-EASL conference. European Association for the Study of the Liver. J Hepatol 2001;35:421-430

27. Bruix J, Sherman M; Practice Guidelines Committee, American Association for the Study of Liver Diseases. Hepatology 2005;42:1208-1236 
28. Sangiovanni A, Manini MA, Iavarone M, et al. The diagnostic and economic impact of contrast imaging techniques in the diagnosis of small hepatocellular carcinoma in cirrhosis. Gut 2010;59:638-644

29. Bruix J, Sherman M. American Association for the Study of Liver Disease. Management of hepatocellular carcinoma: an update. Hepatology 2011;53:1020-1022

30. Bruno S, Di Marco V, Iavarone M, et al. Survival of patients with HCV cirrhosis and sustained virological response is similar to the general population. J Hepatol 2016;64:1217-1223

31. Bruno S, Di Marco V, Iavarone M, et al. Improved survival of patients with hepatocellular carcinoma and compensated cirrhosis who attained sustained virological response. Liver Int 2017;37:1526-1534.

32. Omata M, Cheng AL, Kokudo N, et al. Asia-Pacific clinical practice guidelines on the management of hepatocellular carcinoma: a 2017 update. Hepatol Int 2017;11:317-370

33. Terrault NA, Hassanein TI. Management of the patient with SVR. J Hepatol. 2016;65:S120-9

34. Hepatitis C guidance: AASLD-IDSA recommendations for testing, managing, and treating hepatitis C. www.hcvguidelines.org accessed on July 24, 2017

35. Arase Y, Kobayashi M, Suzuki F, et al. Effect of type 2 diabetes on risk for malignancies includes hepatocellular carcinoma in chronic hepatitis C. Hepatology 2013;57:964-973

36. El Serag HB, Kanwal F, Richardson P, Kramer J. Risk of hepatocellular carcinoma after sustained virological response in veterans with hepatitis $\mathrm{C}$ virus infection. Hepatology 2016;64:130-137

37. Nahon P, Bourcier V, Layese R, et al. Eradication of hepatitis C virus infection in patients with cirrhosis reduces risk of liver and non-liver complications. Gastroenterology 2017;152:142-156

38. Toyoda H, Tada T, Tachi Y, et al. Liver fibrosis indices for identifying patients at low risk of developing hepatocellular carcinoma after eradication of HCV. Antivir Ther 2017;22:185-193 
39. Thandassery RB, Al Kaabi S, Soofi ME, Tharian B, Singh R. Noninvasive serum models to predict significant liver related events in chronic hepatitis C. Hepatol Int 2017;11:401-408

40. Regev A, Berho M, Lennox JJ, et al. Sampling error and intraobserver variation in liver biopsy in patients with chronic HCV infection. Am J Gastroenterol 2002;97:2614-2618

41. Lemon SM, McGivern DR. Is hepatitis C virus carcinogenic? Gastroenterology 2012;142:1274-1278 
Table 1. Baseline demographic and clinical features of the patients according to fibrosis stage (F4 vs. $<\mathrm{F} 4$ )

\begin{tabular}{|c|c|c|c|c|}
\hline Features & $\begin{array}{c}\text { Overall } \\
(n=38)\end{array}$ & $\begin{array}{c}\text { Non-Regressors (F4) } \\
(\mathrm{n}=15)\end{array}$ & $\begin{array}{c}\text { Regressors }(<F 4) \\
(n=23)\end{array}$ & p-value \\
\hline Age, years * & $66(46-75)$ & $65(56-72)$ & $66(46-75)$ & 0.98 \\
\hline Males, $\mathrm{n}$ & $24(65 \%)$ & $9(60 \%)$ & $15(63 \%)$ & 1.0 \\
\hline $\mathrm{BMI}, \mathrm{Kg} / \mathrm{m}^{2} *$ & $24.5(19.9-34.3)$ & $24.9(19.9-34.3)$ & $24.5(20.1-30.4)$ & 0.71 \\
\hline Anti-HBc, $n$ & $17(48.5 \%)$ & $9 / 14(64 \%)$ & $8 / 21(38 \%)$ & 0.18 \\
\hline Diabetes, $\mathrm{n}$ & $4(10.5 \%)$ & $2(13 \%)$ & $2(9 \%)$ & 1.0 \\
\hline Disease duration, months * & $186(60-633)$ & $131(60-449)$ & $215(60-633)$ & 0.75 \\
\hline METAVIR F0/F1/F2/F3/F4, n & $\underline{0 / 2 / 7 / 14 / 15}$ & $\underline{0 / 0 / 0 / 0 / 15}$ & $\underline{0 / 2 / 7 / 14 / 0}$ & $\leq 0.0001$ \\
\hline TE value, $\mathrm{kPa} *$ & $9.8(4.4-34.3)$ & $12.9(7.0-31.6)$ & $9.1(4.4-34.3)$ & 0.004 \\
\hline PLT, $10^{3} / \mathrm{mm}^{3} *$ & $202(85-401)$ & $200(110-283)$ & $206(85-401)$ & 0.75 \\
\hline $\mathrm{ALT}, \mathrm{U} / \mathrm{l} *$ & $21(9-53)$ & $20(9-53)$ & $22(12-47)$ & 0.70 \\
\hline Normal ALT, $\mathrm{n}$ & $27(71 \%)$ & $10(67 \%)$ & $17(74 \%)$ & 0.74 \\
\hline$\gamma \mathrm{GT}, \mathrm{U} / \mathrm{l} *$ & $28(11-109)$ & $36(11-99)$ & $23(13-109)$ & 0.62 \\
\hline Albumin, mg/dl * & $4.6(3.7-5.3)$ & $4.6(3.7-5.1)$ & $5.0(4.4-5.3)$ & 0.10 \\
\hline INR * & $1.01(0.86-1.10)$ & $1.0(0.87-1.10)$ & $1.0(0.86-1.1)$ & 0.98 \\
\hline Bilirubin, $\mathrm{mg} / \mathrm{dl} *$ & $0.5(0.4-2.4)$ & $0.5(0.3-1.3)$ & $1.0(0.3-2.4)$ & 0.71 \\
\hline Cholesterol, $\mathrm{mg} / \mathrm{dl} *$ & $210(154-265)$ & $206(169-258)$ & $215(154-265)$ & 0.46 \\
\hline Cholesterol > $200 \mathrm{mg} / \mathrm{dl}, \mathrm{n}$ & $21(55 \%)$ & $8(53 \%)$ & $13(57 \%)$ & 1.0 \\
\hline $\mathrm{HDL}<60 \mathrm{mg} / \mathrm{dl}, \mathrm{n}$ & $23(61 \%)$ & $9(60 \%)$ & $14(61 \%)$ & 1.0 \\
\hline Triglycerides, mg/dl * & $112(13-211)$ & $110(61-211)$ & $113(13-200)$ & 0.23 \\
\hline Triglycerides $>150 \mathrm{mg} / \mathrm{dl}, \mathrm{n}$ & $5(13 \%)$ & $4(27 \%)$ & $1(4 \%)$ & 0.07 \\
\hline Glucose, $\mathrm{mg} / \mathrm{dl} *$ & $89(71-297)$ & $91(71-297)$ & $86(71-149)$ & 0.08 \\
\hline
\end{tabular}

* Median (range)

BMI: body mass index; TE: transient elastography; PLT: platelets; ALT: alanine amino-transferase; $\gamma \mathrm{GT}$ : gamma-glutamil transferase; INR: international normalized ratio; HDL: high density lipoprotein cholesterol; LB: liver biopsy; HCC: hepatocellular carcinoma 
Table 2. Baseline demographic and clinical features of patients according to HCC development

\begin{tabular}{|c|c|c|c|}
\hline Features & $\begin{array}{l}\text { HCC } \\
(n=5)\end{array}$ & $\begin{array}{c}\text { No HCC } \\
(n=33)\end{array}$ & p-value \\
\hline Age, years * & $65(55-70)$ & $66(46-75)$ & 0.48 \\
\hline Males, $\mathrm{n}$ & $4(80 \%)$ & $20(61 \%)$ & 0.63 \\
\hline BMI, $\mathrm{Kg} / \mathrm{m}^{2} *$ & $25.0(24.6-27.6)$ & $25.3(19.9-34.3)$ & 0.87 \\
\hline Anti-HBc, $n$ & $3(60 \%)$ & $14(42 \%)$ & 0.64 \\
\hline Diabetes, $\mathrm{n}$ & $2(40 \%)$ & $2(6 \%)$ & 0.07 \\
\hline Disease duration, months $*$ & $189(54-237)$ & $183(6 .-633)$ & 0.91 \\
\hline TE value, $\mathrm{kPa} *$ & $12.6(5.7-34.3)$ & $9.8(4.4-31.6)$ & 0.78 \\
\hline PLT, $\mathrm{mm}^{3} *$ & $193(85-313)$ & $204(103-401)$ & 0.91 \\
\hline ALT, $\mathrm{U} / 1 *$ & $20(10-46)$ & $22(9-53)$ & 0.88 \\
\hline Normal ALT, $\mathrm{n}$ & $4(67 \%)$ & $23(72 \%)$ & 1.0 \\
\hline$\gamma \mathrm{GT}, \mathrm{U} / 1 *$ & $74(27-109)$ & $23(11-99)$ & 0.04 \\
\hline Albumin, mg/dl * & $4.7(3.7-5.3)$ & $4.6(3.7-5.2)$ & 0.50 \\
\hline INR * & $1.03(1.0-1.08)$ & $1.0(0.86-1.1)$ & 0.62 \\
\hline Bilirubin, $\mathrm{mg} / \mathrm{dl} *$ & $0.7(0.5-0.8)$ & $0.5(0.3-2.5)$ & 0.62 \\
\hline Cholesterol, $\mathrm{mg} / \mathrm{dl} *$ & $215(180-234)$ & $206(154-265)$ & 0.19 \\
\hline Cholesterol > $200 \mathrm{mg} / \mathrm{dl}, \mathrm{n}$ & $3(60 \%)$ & $18(55 \%)$ & 1.0 \\
\hline $\mathrm{HDL}<60 \mathrm{mg} / \mathrm{dl}, \mathrm{n}$ & $4(80 \%)$ & $19(58 \%)$ & 0.63 \\
\hline Triglycerides, mg/dl $*$ & $120(93-166)$ & $106(13-211)$ & 0.49 \\
\hline Triglycerides $>150 \mathrm{mg} / \mathrm{dl}, \mathrm{n}$ & $1(20 \%)$ & $4(12 \%)$ & 0.53 \\
\hline Fasting glucose, $\mathrm{mg} / \mathrm{dl} *$ & $99(85-297)$ & $88(71-220)$ & 0.12 \\
\hline Fasting glucose $>126 \mathrm{mg} / \mathrm{dl}, \mathrm{n}$ & $2(40 \%)$ & $2(6 \%)$ & 0.07 \\
\hline
\end{tabular}

* Median (range)

HCC: hepatocellular carcinoma; BMI: body mass index; TE: transient elastography; PLT: platelets; ALT: alanine amino-transferase; $\gamma$ GT: gamma-glutamil transferase; INR: international normalized ratio; HDL: high density lipoprotein cholesterol; LB: liver biopsy; HCC: hepatocellular carcinoma 
Table 3. Baseline variables associated with $\mathrm{HCC}$ development at univariate analysis

\begin{tabular}{lccc}
\hline Features & HR & 95\% C.I. & p-value \\
\hline$\gamma$ GT, U/1 & 1.03 & $1.00-1.06$ & 0.014 \\
Fasting glucose, mg/dL & 1.02 & $1.00-1.02$ & 0.012 \\
Diabetes & 5.62 & $0.93-33.9$ & 0.06 \\
Steatosis & 0.99 & $1.05-0.09$ & 0.09 \\
Forns Score $>6.9$ & 12.8 & $1.14-143.9$ & 0.039 \\
Lok Index $>0.5$ & 6.24 & $1.03-37.6$ & 0.046 \\
PLF $>2.98$ & 19.3 & $1.72-217.6$ & 0.016 \\
\hline
\end{tabular}

HCC: hepatocellular carcinoma; $\gamma \mathrm{GT}$ : gamma-glutamil transferase; PLF: predictive liver fibrosis 
Table 4. Baseline histological features of patients according to HCC development

\begin{tabular}{|c|c|c|c|}
\hline Characteristic & $\begin{array}{l}\text { HCC } \\
(n=5)\end{array}$ & $\begin{array}{c}\text { No HCC } \\
(n=33)\end{array}$ & p-value \\
\hline Core specimen, $\mathrm{mm} *$ & $30(25-30)$ & $30(10-50)$ & 0.36 \\
\hline Fibrosis stage (METAVIR), n & & & 0.06 \\
\hline 0 & 0 & 0 & \\
\hline 1 & 0 & $2(6 \%)$ & \\
\hline 2 & 0 & $7(21 \%)$ & \\
\hline 3 & $3(60 \%)$ & $11(33 \%)$ & \\
\hline 4 & $2(40 \%)$ & $13(40 \%)$ & \\
\hline Activity (METAVIR), $\mathrm{n}$ & & & 0.34 \\
\hline 0 & $4(80 \%)$ & $28(85 \%)$ & \\
\hline 1 & $1(20 \%)$ & $5(15 \%)$ & \\
\hline 2 & 0 & 0 & \\
\hline Portal inflammation, $\mathrm{n}$ & & & 0.06 \\
\hline 0 & $1(20 \%)$ & $12(36 \%)$ & \\
\hline 1 & $3(60 \%)$ & $11(33 \%)$ & \\
\hline 2 & $1(20 \%)$ & $9(27 \%)$ & \\
\hline 3 & 0 & $1(4 \%)$ & \\
\hline 4 & 0 & 0 & \\
\hline Steatosis $>5 \%, \mathrm{n}$ & $2(40 \%)$ & $4(12 \%)$ & 0.17 \\
\hline Steatosis, $\mathrm{n}$ & & & 0.36 \\
\hline$<5$ & $3(60 \%)$ & $29(88 \%)$ & \\
\hline $5-33$ & $1(20 \%)$ & $4(12 \%)$ & \\
\hline $34-66$ & 0 & 0 & \\
\hline$>66$ & $1(20 \%)$ & 0 & \\
\hline Area of fibrosis, $\% *$ & $2.4(1.7-5.9)$ & $2.3(0.6-15.1)$ & 0.48 \\
\hline$\Delta$ area of fibrosis, $\% * \pm$ & $63.5(37.2-75.4)$ & $74.4(-84.1-93.8)$ & 0.38 \\
\hline \multicolumn{4}{|l|}{ Immunohistochemistry, $\mathrm{n}(\%){ }^{\#}$} \\
\hline CK7 IHBCs & $1(20)$ & 0 & 0.13 \\
\hline CK7 HPCs & $4(80)$ & $23(70)$ & 1.0 \\
\hline GS & $5(100)$ & $33(100)$ & 1.0 \\
\hline CD34 & $5(100)$ & $30(91)$ & 1.0 \\
\hline$\alpha \mathrm{SMA}$ & $2(40)$ & $14(45)$ & 1.0 \\
\hline CYP2E1 & $2(40)$ & $7(25)$ & 0.59 \\
\hline
\end{tabular}

* Median (range)

${ }^{ \pm} \Delta$ area of fibrosis: calculate as the difference between baseline (post-SVR) LB and pre-treatment LB area of fibrosis (18)

\# Immunostaining expressed as presence ( $>0$ ) at LB: CD34 available in 37, $\alpha$ SMA in 36, CYP2E1 (>1) in 33 patients

HCC: hepatocellular carcinoma; CK7: Cytokeratin 7; IHBCs: Intermediate hepatobiliary cells; HPCs: Hepatic progenitor cells; GS: Glutamine synthetase; $\alpha$ SMA: anti-smooth muscle actin; CYP: Cytochrome P 
Table 5. Baseline NIT values according to HCC development

\begin{tabular}{lccc}
\hline Non invasive test & $\begin{array}{c}\text { HCC } \\
(\mathbf{n = 5})\end{array}$ & $\begin{array}{c}\text { No HCC } \\
(\mathbf{n = 3 3})\end{array}$ & p-value \\
\hline APRI* & $0.3(0.2-0.9)$ & $0.3(0.2-0.8)$ & 0.65 \\
APRI $>1.5$ & 0 & 0 & 1.00 \\
CDS & $5.0(3.0-6.0)$ & $5.0(3.0-7.0)$ & 0.93 \\
CDS $>8$ & 0 & 0 & 1.00 \\
FIB-4 & $2.1(1.0-2.4)$ & $1.7(0.8-3.7)$ & 0.87 \\
FIB-4 >3.25 & 0 & $2(6 \%)$ & 1.00 \\
FIBRO-Q & $4.0(2.0-6.8)$ & $3.8(1.5-7.9)$ & 0.65 \\
FIBRO-Q $>2.6$ & $4(80 \%)$ & $25(76 \%)$ & 1.00 \\
Forns Score* & $6.5(4.5-8.5)$ & $4.9(4.0-8.0)$ & 0.19 \\
Forns Score $>6.9 *$ & $2(50 \%)$ & $3(10 \%)$ & 0.09 \\
GUCI & $0.4(0.3-0.9)$ & $0.4(0.2-0.9)$ & 0.62 \\
GUCI >0.26 & $4(80 \%)$ & $25(76 \%)$ & 1.00 \\
King's & $7.6(5.9-16.9)$ & $7.7(3.8-20.6)$ & 0.68 \\
King's $>16.7$ & $1(20 \%)$ & $2(6 \%)$ & 0.35 \\
Lok Index & $0.5(0.1-0.6)$ & $0.4(0.1-0.7)$ & 0.31 \\
Lok Index $>0.5$ & $3(60 \%)$ & $5(15 \%)$ & 0.05 \\
PLF** & $2.8(2.2-5.0)$ & $2.6(1.7-4.7)$ & 0.35 \\
PLF $>2.98 *$ & $2(50 \%)$ & $2(10 \%)$ & 0.06 \\
ELF** & $9.4(8.6-9.6)$ & $8.4(6.8-10.0)$ & 0.08 \\
\hline
\end{tabular}

Results are expressed as median values (range) or $\mathrm{n}(\%)$

* Calculated in 4 patients with HCC and 29 patients w/o HCC; ** Calculated in 4 patients with HCC and 25 patients w/o HCC.

NIT: non invasive test; HCC: hepatocellular carcinoma. 


\section{Supplementary Table 1. NIT formula}

\begin{tabular}{|c|c|}
\hline $\begin{array}{l}\text { APRI } \\
\text { AST to Platelet Ratio }\end{array}$ & AST levels $\left(\mathrm{x}\right.$ ULN)/platelets count $\left(10^{3} / 1\right)$ x 100 \\
\hline $\begin{array}{l}\text { CDS } \\
\text { Cirrhosis Discriminate Score }\end{array}$ & $\begin{array}{l}\text { Calculated by summing the scores awarded for the following laboratory } \\
\text { results } \\
\text { INR: } 0<1.1 ; 11.1-1.4 ; 2>1.4 \\
\text { ALT/AST: } 0>1.7 ; 11.7-1.2 ; 21.19-0.6 ; 3<0.6 \\
\text { PLT/mm } 0>340 ; 134 .-280 ; 2279-220 ; 3219-160 ; 4 \text { 159-100; } 5 \text { 99-40; } 6 \\
<40\end{array}$ \\
\hline FIB-4 & [age (yr) x AST (U/l)]/[PLT $\left.\left(10^{9} / 1\right)\right] \times$ [ALT (U/l)1/2] \\
\hline FibroQ & {$[(10 \times$ age $(\mathrm{yr})) \times \mathrm{AST}(\mathrm{U} / \mathrm{l}) \times \mathrm{PT}$ INR $] /\left[\operatorname{PLT}\left(10^{9} / \mathrm{l}\right) \times \operatorname{ALT}(\mathrm{U} / \mathrm{l})\right]$} \\
\hline Forns Score & $\begin{array}{l}7.811-3.131 \times \ln \left[\mathrm{PLT}\left(10^{9} / \mathrm{l}\right)\right] \times 0.781 \ln [\gamma \mathrm{GT}(\mathrm{U} / \mathrm{l})]+3.467 \times \ln [\text { age } \\
(\mathrm{yr})]-0.014[\text { cholesterol }(\mathrm{mg} / \mathrm{dl})]\end{array}$ \\
\hline $\begin{array}{l}\text { GUCI } \\
\text { Goteborg University Cirrh }\end{array}$ & $\left(\right.$ AST/ULN) $\times$ INR $\times 100 /$ PLT $\left(10^{9} / 1\right)$ \\
\hline King Score & Age (yr) x AST (U/l) x INR/PLT $\left(10^{9} / 1\right)$ \\
\hline Lok Index & $-5.56-0.0089 \times$ PLT $\left(10^{3} / \mathrm{mm}\right)+1.26 \times$ AST/ALT ratio $+5.27 \times$ INR \\
\hline PLF & $0.956+0.084 \times$ TE -0.004 x King Score $+0.124 \times$ Forns Score $+0.202 \times$ \\
\hline Predicted Liver Fibrosis & APRI score \\
\hline $\begin{array}{l}\text { ELF } \\
\text { Enhanced Liver Fibrosis }\end{array}$ & $\begin{array}{l}-7.412+\left[\ln (\mathrm{HA}) \_0.681\right]+[\ln (\mathrm{PIIINP})-0.775]+[\ln (\mathrm{TIMP}-1)-0.494]+ \\
10\end{array}$ \\
\hline
\end{tabular}

AST: aspartate aminotransferase; ULN: upper limit of normal; INR: International Normalized Ratio; ALT: alanine aminotransferase; PLT: platelets; yr: years; PT: protrombin time; $\gamma \mathrm{GT}$ : gamma-glutamil transferase; TE: transient elastography; HA: hyaluronic acid; PIIINP: N-terminal propeptide of type III collagen; TIMP: tissue inhibitor of metalloprotease 
Supplementary Table 2. Baseline NITs \# according to post-SVR fibrosis stage and reference cut-offs for the diagnosis of cirrhosis 24

\begin{tabular}{|c|c|c|c|c|c|}
\hline Test & $\begin{array}{c}\text { Reference } \\
\text { Cut-off for F4 }\end{array}$ & $\begin{array}{l}\text { Overall } \\
(n=38)\end{array}$ & $\begin{array}{c}\text { Regressors } \\
(n=23)\end{array}$ & $\begin{array}{l}\text { Non-Regressors } \\
\qquad(\mathrm{n}=15)\end{array}$ & p-value \\
\hline APRI & $\geq 1.5$ & $0.3(0.2-0.9)$ & $0.3(0.2-0.9)$ & $0.3(0.2-0.8)$ & 0.48 \\
\hline CDS & $>8$ & $5(2-7)$ & $5(2-6)$ & $5(3-7)$ & 0.49 \\
\hline FIB-4 & $>3.25$ & $1.7(0.8-3.7)$ & $1.7(0.8-2.7)$ & $1.7(1.1-3.7)$ & 0.38 \\
\hline FibroQ & $>2.6$ & $3.9(1.5-7.9)$ & $3.9(1.5-6.3)$ & $4.0(1.7-7.9)$ & 0.26 \\
\hline Forns Score* & $>6.9$ & $5.1(3.1-8.5)$ & $5.1(3.1-8.5)$ & $5.3(4.0-8.0)$ & 0.83 \\
\hline GUCI Index & $>0.26$ & $0.4(0.2-1.0)$ & $0.4(0.18-0.95)$ & $0.4(0.21-0.90)$ & 0.56 \\
\hline King Score & $>16.7$ & $7.7(3.8-20.6)$ & $7.7(3.8-16.9)$ & $7.7(4.7-20.6)$ & 0.48 \\
\hline Lok Index & $>0.5$ & $0.4(0.1-0.7)$ & $0.4(0.1-0.6)$ & $0.4(0.1-0.7)$ & 0.24 \\
\hline PLF* & $>2.98$ & $2.5(1.7-5.0)$ & $2.5(1.7-5.0)$ & $2.5(2.2-4.7)$ & 0.01 \\
\hline ELF** & $\begin{array}{l}>9.3,>9.8, \\
>10.3,>11.3\end{array}$ & $8.6(6.8-10.0)$ & $8.6(7.0-10.0)$ & $8.4(6.8-9.9)$ & 0.70 \\
\hline
\end{tabular}

\# Results are reported as median (range) values

* Calculated in 33 patients for whom valid TE assessments and/or cholesterol values were available ** Calculated in 29 patients 